\title{
On Relating Some Models for Concurrency
}

\author{
Pierpaolo Degano* Roberto Gorrieri ${ }^{\dagger} \quad$ Sebastiano Vigna ${ }^{\ddagger}$
}

\section{Introduction}

The activities of concurrent distributed processes are commonly considered as events that are atomic and occur instantaneously; they often carry a label, indicating the kind of activity performed. Instead, many different, ways are proposed in the literature to compose and organize events in order to represent the runs of a process, i.e., its behaviour. A coarse classification divides these descriptions according to two orthogonal axes: interleaving vs. causality-based representations, and linear vs. branching time ones. In the interleaving models, the events of a run simply occur one after the other, and there is no way to express whether there is any causal link between them, due, e.g., to some shared resource or because they happen in the same place. The causality-based representations generally use a partial ordering relation on the events in order to reflect these potential causal dependencies and independencies. Once chosen the favourite representation of the runs of a process, its behaviour is obtained by giving them some structure. When these descriptions form a set, we get the so-called linear time models. In the branching time models, the representation of process runs are organized as a tree by identifying those events shared by different runs, thus making explicit when a choice occurred.

In order to exemplify, we mention Hoare's traces [Hoa85] as an example of interleaving, linear time model: a process behaviour is a language, the words of which are sequences of labels. In this way the temporal (or generation) ordering in which the events occur is recorded by the sequence of their labels. Milner's synchronization trees [Mils0] are an example of interleaving, branching time model; they are trees with labelled arcs, that represent event occurrences, so the choices and the generation ordering are explicitly represented. Partial orderings of (labelled) events are quite popular a causality-based linear time model (see, e.g., Pratt's pomsets [Pra.86]). In these models, the causal dependencies are explicilly represented by the partial ordering relation, and absence of ordering is interpreted as causal independence. The most studied causality-based, branching time model are (labelled) events structures [Wins7] that are essentially partial orderings of events plus an additional relation, called conflict: two events are in conflict if the occurrence of one of them in a run excludes the other to occur in the same run.

*Dipartimento di Matematica, Università di Parma, Via M. d'Azeglio 85, I-43100 Parma PR, Italy, Fax: +39-521-902350, and Dipartimento di Informatica, Università di Pisa, Corso Italia 40, 1-56125 Pisa PI, Italy, Fax: +39-50-510226; e.mail: degano@di.unipi.it.

tDipartimento di Matematica, Università di Bologna, Piazza di Porta San Donato 5, I-40127 Bologna BO, Italy, Fax: +39-51-354490; e_mail: gorrieri@cs.unibo.it.

${ }^{\ddagger}$ Dipartimento di Scienze dell'Informazione, Università di Milano, Via Comelico 39/41, I-20135 Milano MI, Italy, Fax: +39-2-55006276; e_mail: vigna@ghost.sm.dsi.unimi.it. 
There are no absolute criteria to prefer one among the above models. Indeed, the choice of one of them depends on which aspects of processes are of interest, on the mathematical and mechanical tools available for analysing process behaviour, and also on personal taste. This paper aims at making the choice a little bit less arbitrary, by formally relating the four classes arising from the combination of interleaving or causality-based models with the linear or branching time ones. Intuitively, it is easy to compare these classes, but only few papers address formally this issue, among the others [SNW, Roz90]. Also, establishing formal relationships reveals some subile differences belween causality-based models.

For example, you may go from a branching time representation to a linear time representation of a process just by taking the "path language" of the former. Similarly, it may seem easy to go from a partial ordering of events to a set of traces, by taking all the total orderings, i.e., all the sequences of (labels of) events that are compatible with the partial ordering (containing $i t$, in set-theoretical terms). However, this is not always the case, because the absence of ordering between two events does not necessarily imply that they may occur in any temporal order, although this is quite common an assumption (e.g., see Kahn'-Plotkin's $Q$ axiom, the semantics of asynchronous composition in CCS and similar languages, the representation theorem of prime event structures as domains of configurations). Indecd, a relation of priorily, both on conflicting and on causally independent cvents, has been recently advocated [JGS8] in order to compactly specify the behaviour of processes in critical situations, e.g., to prevent faults or to cope with them. Therefore, it may well happen that lwo causally unrelated events may occur only in a fixed temporal ordering.

Thus, partial orderings of events and event structures are not fully satisfactory when priorities are of interest, while (not surprisingly!) causal trees [DD90] are such. Recall that causal trees are arc-labelled trees wilh enriched labels that, beside the performed activity, supply also indication of its causes. As a matter of fact, causal trees extend synchronization trees with a relation of causality (and the generation ordering is inherited), while event structures extend synchronization trees with a relation of causal independence, with no regard to the generation ordering $(c f$. how a prime event structure is recovered from a domain of configurations).

Our main contribution is a first step towards a lormal characterization of which information is lost when passing from the causality-based, branching time models to the interleaving, branching time and the causality-based, linear time ones, and from these to the interleaving, linear time models. Said in the other way around, we establish which is the necessary and sufficient information that enables us to reconstruct the causalitybased, branching time behaviour of a process from those behaviours lacking the information about the causal or about the branching structures, provided that the latter two agree when interpreted in the interleaving, linear time setting.

A more precise synopsis of our paper follows. We chose a representative for each of the four classes of behaviour discussed above, and we present them as four categories: TL, ST, MOL, CT. The objects of the category TL are Hoare's trace languages, while those of ST are Milner's synchrouization trees. Category MOL has the so-called mixed orderings of events as objects [DDM90]. A mixed ordering is a partial ordering of events with an additional total ordering to be interpreted as the generation ordering, that "sequentializes" also the occurrences of events not causally related. The fourth category C'T has causal trees as objects. Actually, each run of a process is indexed and so is its representation. E.g., an object of TL is an indexed set of traces, rather than a language. The morphisms of 
these categories preserve to some extent the structure of their objects. There are obvious forgetful functors from CT to ST and MOL, and from these to TL, that always have left and right adjoints. For example, the left adjoint to the forgetful functor from ST to TL makes the trivial synchronization tree that has a branching only at its root, while the right adjoint glues the traces as much as possible. The forgetful functors have also an axiomatic characterization. The main theorem says that the four categories above form a pullback. More accurately, CT is a reflective and coreflective subcategory of ST $\times_{T L}$ MOL, because of pathological cases descibed in Section 3. We think that other models for concurrency (such as Mazurkiewicz's traces, Pratt's pomsets [PraS6], Janicki' and Koutny's composets [JK91], Rabinovitch' and Trakhtenbrol's behaviour structures [RT88]) can be recast with minor efforts in our framework by establishing pairs of adjoints, one of which is usually an obvious forgetful functor.

In Section 5, we compare event structures and causal trees. It is easy to pass from an event structure to a causal tree, but this transformation is fully invertible only when causally independent events may occur in any temporal ordering and there is no autoconcurrency (no two causally independent events have the same label). This is mainly because event structures are not fully satisfactory when priority has to be expressed. In order to increase their expressive power, we add to event structures a priority relation, besides the causal and the conflict ones. Of course, priority cannot interfere with causality, but it may dictate which event has to be fired in a set of enabled conflicting ones, and which among enabled concurrent events has to occur first. Prioritized event structures are a conservative extension of prime event structures in that their configurations are finitary, coherent, prime algebraic partial orderings, namely domains. Therefore, it is possible to reconstruct a prime event structure with the same "dynamics" of a prioritized one. Essentially, what is lost is that some events may disappear or may be duplicated, and that the priority links may be transformed into causality links. Eventually, the category of causal trees is shown to be a coreflective sub-category of that of prioritized event structures. Additionally, the coreflection shows that the expressive power of the two models is the same.

The last section briefly introduces denotational operators for prioritized nondeterminism and prioritized asynchrony, in terms of event structures and their counterparts in terms of causal trees. The latter helps in defining a causality-based, branching time operational semantics, given in the sos style, for concurrent languages with priority, omitted here because of lacli of space. The operational and denotational semantics in the other classes are recovered easily, by factoring the causality-based semantics with the obvious counterparts of the axioms characterizing the forgetful functors from CT to ST, MOL, and TL.

\section{Prime Event Structures}

We assume that the reader is familiar with basic domain theory; we only recall that a poset $(P, \leq)$ is a Scott domain if $P$ is consistently complete and algebraic. The order structure we are interested in is a particular kind of Scott domains; it is a finitary, coherent, prime algebraic poset, that we will simply call a domain.

The notion we recall from the literature is that of a prime event structure [NPW81, Win87] (we will often omit the altribute prime). They form a well-studied semantic model 
for true concurrency in the branching-time approach.

An event structure $\mathcal{E}=\langle E, \leq, \#\rangle$ consists of a poset $(E, \leq)$ and an irreflexive relation $\# \subseteq E \times E$ of conflict (disjoint from $\leq$ ) such that

(i) $E$ is a countable set of events,

(ii) for every $e \in E, c$ is finitely preceded, i.e., $\left\{\epsilon^{\prime} \mid e^{\prime} \leq e\right\}$ is finite,

(iii) the conflict relation is hereditary, i.e., $e \# e^{\prime} \leq e^{\prime \prime} \Rightarrow e \# e^{\prime \prime}$, for every $e, e^{\prime}, e^{\prime \prime} \in E$.

One usually calls cvents the elements of $E$ and says that $e^{\prime}$ causes $e$ if $e^{\prime} \leq e$; we use $e \geq e^{\prime}$ iff $e^{\prime} \leq e$. Another derived relation is the concurrency relation $c o=E \times E \backslash(\leq \cup \geq U \#)$ : two events are concurrent if they are not related in either relation.

Given a set of events $X$, the sets of the minimal events, denoted $\min (X)$, is defined as follows:

$$
\min (X)=\left\{e \in X \mid\left(e^{\prime} \in X \wedge e^{\prime} \leq e\right) \Rightarrow e=e^{\prime}\right\},
$$

A subset $X \subseteq E$ is a configuration when it satisfies the following conditions:

- $X$ is a cone, i.e., $\forall x \in X . \forall y \in E . y \leq x \Rightarrow y \in X$.

- $X$ is conflict-free, i.e., $X \times X \cap \#=\emptyset$.

$\operatorname{Conf}(\mathcal{E})$ denotes the set of configurations of $E$.

Another way of characterizing a cone is through the notion of securing sequence. An event $e$ is secured in $X$ if there exists a sequence $e_{0} e_{1} \ldots e_{n}$ of events in $X$ such that $e_{n}=e$ and $e_{i} \in \min \left(E \backslash\left\{e_{0}, \epsilon_{1}, \ldots e_{i-1}\right\}\right)$. A set $X$ is a cone if for all $e$ in $X, e$ is secured in $X$.

Since a principal configuration $\downarrow \epsilon=\left\{e^{\prime} \in E \mid e^{\prime} \leq e\right\}$ is contained into another configuration $X$ if and only if $e \in X$, every configuration is the union $(=\sup$ in $\operatorname{Conf}(\mathcal{E})$ ) of the principal configurations contained in it. When ordered by inclusion, $\operatorname{Conf}(\mathcal{E})$ is a domain, where the complete primes are the principal configurations. We are now in a position to state the representation theorem for prime event structures [NPWS1].

Theorem 2.1 If $\mathcal{E}$ is a prime event structure, then $\operatorname{Conf}(\mathcal{E})$ is a domain. Moreover any domain $\mathcal{D}$ is of the jorm $\operatorname{Conf}(\mathcal{E})$ for some evcnt structure $\mathcal{E}$.

The event structure $\mathcal{E}$ mentioned in the stalement above is built on the set of complete prime elements of $\mathcal{D}$, with the induced order and with the conflict relation defined by $p \# q$ if $\neg \exists x \in \mathcal{D}(p \leq x \wedge q \leq x)$. Note that two events are set concurrent if and only if they may occur in any order.

When events carry labels over an alphabet $\Sigma$, we obtain labelled event structures, i.e., quadruples of the form $\mathcal{E}=\langle E, \leq, \#, \ell\rangle$, where $\ell: E \rightarrow \Sigma$.

\section{Some useful categories}

In this section we describe some categories which we will use in order to relate some classical models of concurrency. As anticipated in the introduction, we will consider a representalive for each class of process behaviour arising from the combination of the interleaving/causality-based and linear/branching time parameters. Hoare's traces are the representative for interleaving, linear time models, and describe the behaviour of a process 
as a set of sequences of event labels. Then, synchronization trees are considered for the interleaving, branching time case. Adding a causality relation on the sequence of events of a run yields mixed ordering of events, the representative of causality-based, linear time models. Finally, we consider causal trees, that are synchronization trees enriched with a causality information as our last model. Actually, in any model we index the runs of a process, in order to distinguish those that are represented in the same way. For example, a process that may perform in two different runs the same sequence of events labelled, say, $\alpha$ and $\beta$ is rendered in our interleaving, linear representative as $\{\langle 0, \alpha \beta\rangle,\langle 1, \alpha \beta\rangle\}$. Similarly, the two mixed orderings of events it originates carry indexes. In the branching time representatives, indexes are attached to paths of trees. We assume in the sequel a fixed alphabet $\Sigma$. The letter $J$, possibly with subscripts, will be always used to denote sets of indexes.

We first give a categorical structure to multi-sets of Hoare's traces.

Definition 3.1 The catcgory TL of sequences on $\Sigma$ has as objects extent functions $J_{S} \stackrel{S}{\longrightarrow}$ $\Sigma^{*}$. Morphisms from $S$ to $S^{\prime}$ are given by maps

$$
f: J_{S} \longrightarrow J_{S^{\prime}}
$$

such that $S(j)$ is a prefix of $S^{\prime \prime}(f(j))$.

The next definition is a categorical version of synchronization trees, defined on top of traces by providing a "glueing" function that iclentifies part of the common prefix of two traces.

Definition 3.2 The category ST of synchronization trees labelled on $\Sigma$ has as objects pairs $T=\left\langle e_{T}, g_{T}\right\rangle$, where $e_{T}$ is an extent function $J_{T} \stackrel{e_{T}}{\longrightarrow} \Sigma^{*}$, and $g_{T}$ is a glueing function $g_{T}: J_{T} \times J_{T} \longrightarrow \Sigma^{*}$ satisfying

$$
\begin{aligned}
g_{T}\left(j_{1}, j_{2}\right) & =g_{T}\left(j_{2}, j_{1}\right) \\
g_{T}\left(j_{1}, j_{2}\right) & \leq e_{T}\left(j_{1}\right), e_{T}\left(j_{2}\right) \\
g_{T}\left(j_{1}, j_{2}\right) \wedge g_{T}\left(j_{2}, j_{3}\right) & \leq g_{T}\left(j_{1}, j_{3}\right) \text { (triangular inequality) }
\end{aligned}
$$

where $\leq$ denotes the prefix ordering and $\wedge$ denoles the respective meet operation.

Morphisms $T \longrightarrow T^{\prime}$ are functions $f: J_{T} \longrightarrow J_{T^{\prime}}$ which increase extent and glueing, i.e.,

$$
\begin{aligned}
e_{T}(j) & \leq e_{T^{\prime}}(f(j)) \\
g_{T}\left(j_{1}, j_{2}\right) & \leq g_{T^{\prime}}\left(f\left(j_{1}\right), f\left(j_{2}\right)\right) .
\end{aligned}
$$

Technically speaking, the above is equivalent to defining a symmetric catcgory enriched over a bicategory, introduced by R.F.C. Walters in [Wal\$2] and applied to concurrency in [KLP90]. However, we will not need the full bicategorical machinery in our treatment. Note also that the morphisms we define are slightly more general than functors: indeed, they are functors into the Cauchy-completion (in the sense of Lawvere [Law74]) of the codomain.

We now recall the notion of labelled mixed orderings of events, and then we define the category of their languages as the representative for the causality-based, linear time 
models. We will use an extended alphabet $\Sigma \times \operatorname{Pfin}(\mathrm{N})$ (where $\operatorname{Pfin}(\mathrm{N})$ denotes the finite parts of the natural numbers): in each label $\left\langle\sigma, K^{\prime}\right\rangle$, the set of natural numbers $K$ specifies the causes of $\sigma$, and it is interpreted as a. set of backwards pointers to the preceding labels. These pointers encode a partial ordering under obvious consistency conditions (they must point to some labels, and they must reflect transitivity of causality).

When dealing with these strings, we will write

$$
\left\langle\sigma_{0}, K_{0}\right\rangle\left\langle\sigma_{1}, K_{1}\right\rangle \cdots\left\langle\sigma_{n}, K_{n}\right\rangle \unlhd\left\langle\sigma_{0}^{\prime}, K_{0}^{\prime}\right\rangle\left\langle\sigma_{1}^{\prime}, K_{1}^{\prime}\right\rangle \cdots\left\langle\sigma_{n^{\prime}}^{\prime}, K_{n^{\prime}}^{\prime}\right\rangle
$$

iff $n \leq n^{\prime}$, and $\sigma_{j}=\sigma_{j}^{\prime}, K_{j} \subseteq K_{j}^{\prime}$. for all $0 \leq j \leq n$.

Definition 3.3 The category MO of mixed ordering labelled on $\Sigma$ has as objects $M$ strings in the alphabet $\Sigma \times \mathrm{Pfin}(\mathrm{N})$ satisfying the following conditions: if

$$
M=\left\langle\sigma_{0}, K_{0}\right\rangle\left\langle\sigma_{1}, K_{1}\right\rangle \cdots\left\langle\sigma_{n}, K_{n}\right\rangle,
$$

then

- for all $p \in K_{j}, 0<p \leq j$ (poinlers are well-defined), and

- if $p \in K_{j}, q \in K_{j-p}$ then $p+q \in K_{j}$ (transitivity of causality).

There is a (unique) morphism $M \longrightarrow M^{\prime}$ if $M \unlhd M^{\prime}$.

This definition is equivalent to specify a finite, totally ordered set which is labelled on $\Sigma$ and endowed with a partial ordering which is contained in the total one. Morphisms are then inclusions which preserve both orders and have a prefix of the target as image.

Note that the preservation of one of the orclers does not imply in general the preservation of the other one. If you consider a map whose source has the trivial partial ordering (i.e., the identity relation), any set map will preserve this ordering, but could not preserve the total one. On the other hand, a map whose target has the trivial partial ordering cannot preserve a non-trivial partial ordering.

Definition 3.4 The category MOL of languages of mixed ordering labelled on $\Sigma$ has as objects functions $J_{O} \stackrel{O}{\longrightarrow} \mathrm{Obj}(\mathrm{MO})$. Morphisms $O \longrightarrow O^{\prime}$ are defined by a function $f: J_{O} \longrightarrow J_{O^{\prime}}$ such that $O(j) \unlhd O^{\prime}(f(j))$.

The representative for causality-based, branching time models we choose is that of causal trees. The present definition is formulated in terms of glueing of mixed orderings.

Definition 3.5 The calegory CT of causal trees labelled on $\Sigma$ has as objects pairs $C=$ $\left\langle e_{C}, g_{C}\right\rangle$, where $e_{C}$ is an extent function $J_{C} \stackrel{e_{C}}{\longrightarrow} \mathrm{Obj}(\mathrm{MOL})$ and $g_{C}$ is a glueing function $g_{C}: J_{C} \times J_{C} \longrightarrow$ Obj(MOL) satisfying

$$
\begin{aligned}
g_{C}\left(j_{1}, j_{2}\right) & =g_{C}\left(j_{2}, j_{1}\right) \\
g_{C}\left(j_{1}, j_{2}\right) & \leq e_{C}\left(j_{1}\right), e_{C}\left(j_{2}\right) \\
g_{C}\left(j_{1}, j_{2}\right) \wedge g_{C}\left(j_{2}, j_{3}\right) & \leq g_{C}\left(j_{1}, j_{3}\right) \text { (triangular inequality) }
\end{aligned}
$$

Morphisms $C \longrightarrow C^{\prime}$ are functions

$$
f: J_{C} \longrightarrow J_{C^{\prime}}
$$


such that

$$
\begin{array}{rll}
e_{T}(j) & \unlhd & e_{T^{\prime}}(f(j)) \\
g_{T}\left(j_{1}, j_{2}\right) & \unlhd & g_{T^{\prime}}\left(f\left(j_{1}\right), f\left(j_{2}\right)\right) .
\end{array}
$$

The above four main calegories are related by a number of forgetful functors, which have right and left adjoints. There is clearly a forgetful functor ST $\longrightarrow$ TL which forgets the glueing information contained in a tree, and a forgetful functor MOL $\longrightarrow$ TL which forgets the causal inlormation. Note that the two causally independent events described by the mixed ordering $\langle\alpha, \emptyset\rangle\langle\beta, \theta\rangle$ originate a single trace $\alpha \beta$, thus reflecting our intuition about priority. Instead, i[ we take partial orderings in place of mixed ones, the two traces $\alpha \beta$ and $\beta \alpha$ are obtained by forgetting the causal relation.

The left adjoint to the forgetful functor ST $\longrightarrow$ TL makes a tree out of a trace language by specifying no glueing, while the right adjoint adds the maximum glueing. Formally,

Proposition 3.1 The forgetful functor $U:$ ST $\longrightarrow$ TL has a right and a left adjoint $R$ and $L$, defined by

$$
\begin{aligned}
e_{L(S)}(j)=e_{R(S)}(j) & =S(j) \\
g_{L(S)}\left(j_{1}, j_{2}\right) & =\varepsilon \\
g_{R(S)}\left(j_{1}, j_{2}\right) & =S\left(j_{1}\right) \wedge S\left(j_{2}\right)
\end{aligned}
$$

The left adjoint to the forgetful functor MOL $\rightarrow$ TL adds no causal information, while the right adjoint adds all the possible causal information. Formally,

Proposition 3.2 The forgetful functor $U:$ MOL $\longrightarrow$ TL has a right and a left adjoint $R$ and $L$. For any object $S \in \mathrm{TL}$, if $S(j)=\sigma_{0} \sigma_{1} \cdots \sigma_{n}$ they are defined by

$$
\begin{aligned}
& L(S)(j)=\left\langle\sigma_{0}, \emptyset\right\rangle\left\langle\sigma_{1}, \emptyset\right\rangle \cdots\left\langle\sigma_{n}, \emptyset\right\rangle \\
& R(S)(j)=\left\langle\sigma_{0}, \emptyset\right\rangle\left\langle\sigma_{1},\{1\}\right\rangle \cdots\left\langle\sigma_{n},\{1,2, \ldots, n\}\right\rangle
\end{aligned}
$$

Causal trees have two trivial forgetful functors on MOL and ST: the first one forgets the conflict information, while the second one forgets the causal information (also this two functor's have obvious left and right adjoints). It is easy to check that we have indeed a commutative diagram

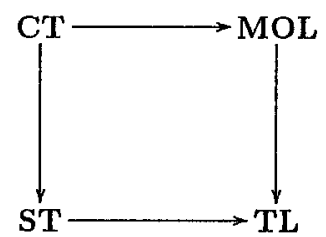

of forgetful functors. The obvious question is if this diagram is a pullback, i.e., if, given a tree (providing conflict information) plus a mixed ordering language (providing causal information) which decribe the same set of paths, we can rebuild uniquely a causal tree that expresses concisely all the information. 
As it stands, the diagran is not a pullback, as the following example shows: take two paths $\langle 0, \alpha \beta \gamma\rangle$ and $\langle 1, \alpha \beta \delta\rangle$ with $g(0,1)=\alpha \beta$. Consider then the mixed ordering language

$$
\{\langle 0,\langle\alpha, \emptyset\rangle\langle\beta, \theta\rangle\langle\gamma, \emptyset\rangle\rangle,\langle 1,\langle\alpha, \emptyset\rangle\langle\beta,\{1\}\rangle\langle\delta, \emptyset\rangle\rangle\} .
$$

There is no obvious way to turn this pair into a causal tree, because the causality information specified by the mixed ordering language contradicts the conflict information given by the tree (indeed, the glueing information of the tree says that the two observation were completely coincident in the first tuo stcps, so that we could not observe different causal relations).

However, the pullback ST $\times$ TL MOL has a strict relation with CT, because the functor $\mathrm{CT} \longrightarrow \mathrm{ST} \times{ }_{\mathrm{TL}}$ MOL induced by the universal property of the pullback sends a causal tree to the pair given by the underlying $\Sigma$-labelled tree, and by the mixed ordering language specified by its extent function. Since a causal tree is completely defined by these data, the mapping is injective.

Moreover, this functor has two adjoints, which deal with pairs of mixed orderings which have a different causal information in the initial segment glued by $g_{C}$. When such a conflict occurs, the right adjoint takes the intersection of the two causal orders. Intuitively, the extra causal information is considered spurious, because another run sharing the same prefix does not show it. On the contrary, the left adjoint takes the least order generated by the union of the two causal orderings. In this case, the extra information is considered valid, as if it were "skipped" while representing one run.

Theorem 3.1 CT is a reflective and corcflective subcategory of ST ×TL MOL.

Note that the two adjoints behave well with respect to objects of $\mathbf{S T} \times \mathbf{T L}$ MOL which are image of the inclusion functor from CT. This condition simply means that no glueing is made of labels of mixed orderings reporting inconsistent information. When this is the case, the left and the right adjoints do coincide, and re-construct a causal tree isomorphic to the original one.

The forgetful functors of the previous commutative diagram can be described algebraically, when trees are represented as algebras generated by the empty tree, prefixing and summation (of course, two new categories should be defined for trees, equivalent to CT and ST). It is well-known that passing from a branching time representation to a linear one is expressed by the axiom

$$
\lambda \cdot\left(Z+Z^{\prime}\right)=\lambda \cdot Z+\lambda \cdot Z^{\prime}
$$

for any kind of label $\lambda$. Of course, when $\lambda \in \Sigma$, we get an algebraic characterization of the forgetful functor $\mathrm{ST} \longrightarrow \mathrm{TL}$, while the one $\mathrm{CT} \longrightarrow \mathrm{MOL}$ is captured when $\lambda \in \Sigma \times \operatorname{Pfin}(\mathbf{N})$. Instead, in order to forget causality one can use the axiom

$$
\left\langle\sigma, K^{\prime}\right\rangle \cdot Z=\left\langle\sigma, K^{\prime}\right\rangle \cdot Z,
$$

that, when $Z$ is instantiated to a (causal) tree, characterizes the forgetful functor CT $\longrightarrow$ $\mathrm{ST}$, and that describes the forgetful functor MOL $\longrightarrow$ TL, when $Z$ is a mixed ordering.

In our setting, it is also straightforward to axiomatize the obvious forgetful functor from mixed orderings to pomsets, that cliscards the generation ordering and enforces 
the assumption that concurrent events may occur in any order. The following equation expresses precisely this fact:

$$
\langle\sigma, K\rangle\left\langle\rho, K^{\prime \prime}+1\right\rangle M=\left\langle\rho, K^{\prime \prime}\right\rangle\langle\sigma, K+1\rangle M .
$$

\section{Adding Priorities to Event Structures}

Nondeterminism is a powerful notion which permits to have compact descriptions of systems with a large number of details which, when taken into account, may turn it into a deterministic systems. Similarly, we see priority as a tool for abstracting from complex relations among events that are visible only when the description of the system is given at a low level. As a matter of fact, these relations have mainly to do with a combination of temporal constraints and a shared use of limited resources. Almost all these details may be ignored when compactly describing a system at a high level, except for those concerning the common resources. Priority gives an obvious discipline for breaking the tie between conflicting events. Instead, when two events are considered concurrent at a high level, priority imposes on them a timing order, but not a causality one; if unfortunately the timing order is violated, the prioritized event will never occur.

As an example, consider Pierpaolo and Roberto who wish to take the same plane, but only the latter has a reservation. So, Roberto has priority over Pierpaolo, who is standing by. If both are at the airport in time and a single seat is free, Roberto gets it. Abstractly, this situation is represented by a prioritized conflict between the events Roberto-flies and Pierpaolo-flies. Instead, if there are seats enough, first Roberto and then Pierpaolo get their places (and no causal relation holds between the two events). If however Roberto is not at the check-in desk in time, while Pierpaolo is there, only the latter is accomodated and the former will never have a. seat on that flight (Sebastiano has wings...).

The main aim of this section is the extension of the model of prime event structures in which a priority relation, i.e. a partial ordering $\ll$ between events is accomodated smoothly. The only constraints on $\ll$ are that it does not interfere with $\leq$ and it should enjoy the property of being a finitely preceding relation. Formally, we have the following.

Definition 4.1 A prioritized labelled event structure (ples, for short) is a quintuple $\mathcal{E}=$ $\langle E, \leq, \#, \ll, \ell\rangle$ where:

- $\mathcal{E}^{\prime}=\langle E, \leq, \#, \ell\rangle$ is an event structure (its associaled prime event structure),

- « is a partial ordering on $E$ such that

(i) $\ll \cap \geq=\{\langle c, e\rangle \mid e \in E\}$,

(ii) for every $e \in E$, $e$ is finitely priorilized, i.e., $\left\{e^{\prime} \mid e^{\prime} \ll e\right\}$ is finite.

According to the above intuition, if $e \ll e^{\prime}$ ( $e$ has priority on $\left.e^{\prime}\right)$ and $e \# e^{\prime}$, whenever both are enabled $e$ is chosen and $e^{\prime}$ is discarcled; if instead only $e^{\prime}$ is enabled, then it may be executed. When $e \ll e^{\prime}$ and $e$ co $e^{\prime}$, whenever both are enabled $e$ is executed first and $e^{\prime}$ is delayed; if instead only $e^{\prime}$ is enabled, then it may be executed, but its occurrence prevents $e$ to be executed, even if enabled later on. Therefore, the configurations of 
prioritized labelled event structures, called pri-configurations, require a stronger version of cone. The additional constraint takes into account the priority relation. Let us first consider two simple examples.

Example 4.1 The ples in Figure 1.a) represents two independent events, $\alpha$ and $\beta$, where $\beta$ has priority over $\alpha$. Its intended poset of pri-configurations is given in $b$ ); note that the unique configuration which is not a pri-one is $\{\alpha\}$. In Figure 1.c) we have another ples; all of its configurations are pri-configuralions, but there is no arc from $\{\gamma\}$ to $\{\alpha, \gamma\}$, due to the priority of $\beta$ over $\alpha$.

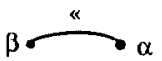

a)

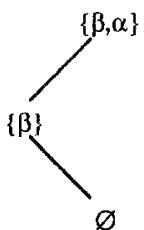

b)

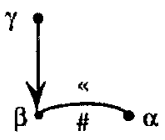

c)

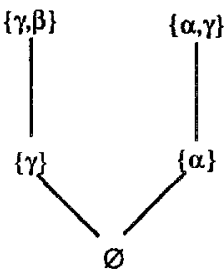

d)

Figure 1: Posets of pri-configurations for two simple ples's

We need an auxiliary definition. Given a ples and a set $X$ of events, let $\operatorname{pri}-\min (X)$ be the set of the events in it which are minimal w.r.t. botls the causality and priority relations, i.e.,

$$
\begin{aligned}
\operatorname{pri}-\min (X)= & \left\{e \in X \mid\left(e^{\prime} \in X \wedge e^{\prime} \leq e\right) \Rightarrow e=e^{\prime}\right\} \cap \\
& \left\{c \in X \mid\left(e^{\prime} \in X \wedge e^{\prime} \ll e\right) \Rightarrow e=e^{\prime}\right\} .
\end{aligned}
$$

Definition 4.2 Given the ples $\varepsilon=\langle E, \leq, \#, \ll, \ell\rangle$, a subset $X \subseteq E$ and an event $e \in X$, we say that $e$ is pri-secured in $X$ if there exisls a scquence (oflcn called a pri-proof) $\epsilon_{0} e_{1} \ldots e_{n}$ of events in $X$ such that $e_{n}=e$ and $e_{i} \in \operatorname{pri-min}\left(E \backslash\left\{e_{0}, e_{1}, \ldots e_{i-1}\right\}\right)$.

As an example, consider the pri-configuration $\{\alpha, \beta\}$ in Figure 1.b); there is a unique pri-securing sequence of $\alpha$ in it, namely $\beta \alpha$. In the pri-configuration $\{\alpha, \gamma\}$ in Figure $1 . d)$, both $\alpha \gamma$ and $\gamma$ are pri-prools of $\gamma$; instead $\gamma \alpha$ is not a pri-proof for $\alpha$. (Note that there is no need to introduce infinite pri-prools, because the causality and priority relations are finitely preceded.)

Definition 4.3 Given the ples $\mathcal{E}=\langle E, \leq, \#, \ll, \ell\rangle$, a subset $X \subseteq E$ is a pri-configuration if the following hold:

(i) $X$ is conflict-free, and

(ii) $\forall e \in X$.e is pri-secured in $X$.

Let $\operatorname{Pconf}(\mathcal{E})$ be the Jamily of the pri-configurations of $\mathcal{E}$. 
Obviously, every pri-configuration of a ples is also a configuration of its associated les. The vice versa does not hold, as shown in Figure 1.b). Also, Figure 1.d) shows that the partial ordering on pri-configurations is contained in set inclusion. Indeed, an event can be added to a pri-configuration $X$, if it belongs to pri-min $(E \backslash X)$ and if, additionally, it has no priority over the elements of $X$.

Definition 4.4 Let $X, X \cup\{e\} \in \operatorname{Pconf}(\mathcal{E})$. Define $X \prec X \cup\{e\}$ if

(i) $e \in \operatorname{pri-\operatorname {min}}(E \backslash X)$ and

(ii) $\forall e^{\prime} \in \operatorname{pri-min}(E \backslash X), e \notin e^{\prime}$.

Finally, let $\sqsubseteq$ be the reflexive, transitive closure of $\prec$, i.e., $\sqsubseteq=\prec *$.

Note that for all pri-configurations $X \in \operatorname{Pconf}(\mathcal{E}), \emptyset \sqsubseteq X$.

Example 4.2 Consider the ples and its poset of configurations depicted in Figure 2. In this case, several configurations (e.g., $\{\beta, \delta\}$ ) are not pri-ones, but $\sqsubseteq=\subseteq$. This is no more the case for the ples in Figure 3. Afler the execution of the event $\rho$, there is only one pri-minimal evcnl, namely $\alpha$, hence $\{\rho\} \nsubseteq\{\beta, \rho\}$. Condition (ii) above makes $\{\beta, \rho\} \nsubseteq\{\beta, \rho, \alpha\}$, because $\alpha$ cannol be extecuted afler the exccution of the less prioritized event $\beta$.
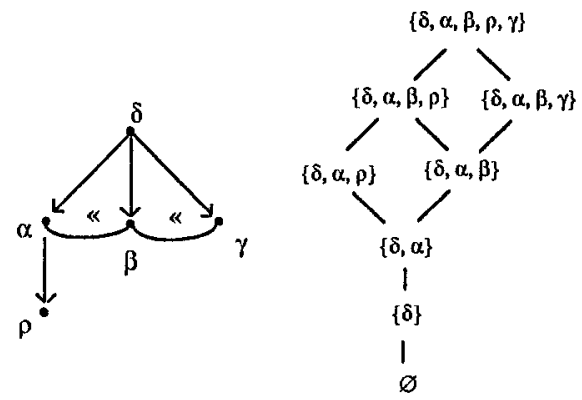

Figure 2: A prioritized event structure and its intended poset of configurations. Note that $\alpha \ll \gamma$, by transitivity of $\ll$.

Theorem 4.1 The partially ordered set $\mathcal{F}=\langle\operatorname{Pconf}(\mathcal{E}), \sqsubseteq\rangle$ is finitary, coherent and prime algebraic, i.e., a domain.

Proof. (Sketch) Coherence is easy to establish, because pri-proofs are preserved under union of compatible configurations. The poset is finitary because both causality and priority relations are finitely preceded. Prime algebraicity requires to resort to the easier fact that $\mathcal{D}=\langle\operatorname{Pconf}(\mathcal{E}), \subseteq\rangle$ is a domain, too. Actually, given a pri-configuration $X$, for every $P$ complete prime in $\mathcal{D}, P \subseteq X$, there exists a $Q$ complete prime in $\mathcal{F}, Q \sqsubseteq X$, such that $P \subseteq Q$. 

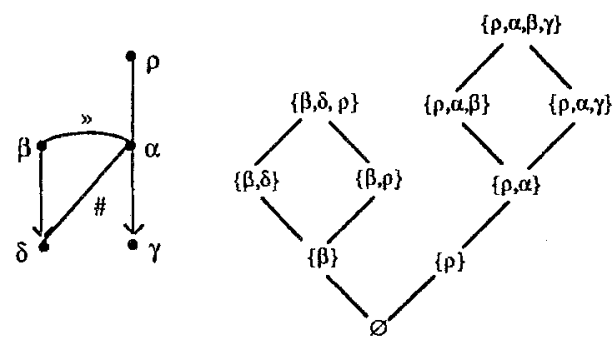

Figure 3: A prionitized event structure and its intended poset of pri-configurations. Note that no arc connects $\{\rho\}$ to $\{\beta, \rho\}$, nor $\{\beta, \rho\}$ to $\{\rho, \alpha, \beta\}$.

The above theorem permits to build a prime event structure from the domain of priconfigurations of a prioritized one, by applying the construction of the representation theorem. However, the relevant information about the causal independence of temporal dependent events is lost. Indeed, from the domain in Figure 1.b), we get the prime event structure in Figure 4.a), in which the priority relation has been tranformed in a causal one. From the domain in Figure 1.d) one obtains the les in Figure 4.b), where the original event $\gamma$ has been duplicated, because $\gamma$ and $\alpha$ cannot occur in any order, although independent (firing $\gamma$ first, excludes $\alpha$ to occur). As last example, consider the les in Figure 4.c), originated from the domain in Figure 3. Note that the original event $\beta$ has been duplicated: one of the new events is in conflict with $\alpha$, the other is caused by $\alpha$.

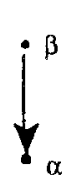

a)

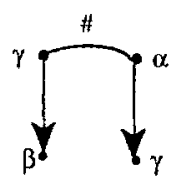

b)

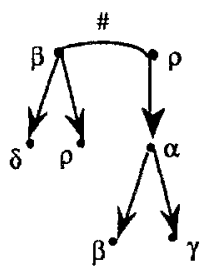

c)

Figure 4: Prime event structures obtained from the domains of pri-configurations of the ples's in Figure 1.b), L.d) and 3, respectively.

\section{$5 \quad$ Relating Causal Trees and Prioritized LES}

It is easy to associate to an event structure a causal tree by suitably labelling the unfolding of its domain of configurations. Here we define an adjunction between the categories of causal trees and prioritized event structures, as defined below.

Definition 5.1 The calcgory PLES has as objects prioritized labelled event structures and as morphisms maps $f: E \longrightarrow E^{\prime}$ which prescrve causality and labelling, and which 
induce a map $\operatorname{Pconf}(\mathcal{E}) \longrightarrow \operatorname{Pconf}\left(\mathcal{E}^{\prime}\right)$ (by direct image) preserving the boltom and $\prec$.

Note that these maps may identify conflicting events with the same label.

Now we turn to the definition of the Unf functor, which unfolds a ples $\mathcal{E}$ to a causal tree: recall that for all $X, X^{\prime} \in \operatorname{Pconf}(\mathcal{E})$, if $X \prec X^{\prime}$ then $X^{\prime}=X \cup\{e\}$. Thus, the set of finite $\prec$-paths starting from the bottom of the domain of pri-configurations gives us the indexing set $J$ of the tree $\operatorname{Unf}(\mathcal{E})$; each index is mapped to the string of labels of the events occurring along the palh paired with backpointers to their causes. The glueing function is given by $g\left(j_{1}, j_{2}\right)=j_{1} \wedge j_{2}$ (i.e., the greatest common subpath of $j_{1}$ and $j_{2}$ ).

Given a ples morphism $f: \mathcal{E} \longrightarrow \mathcal{E}^{\prime}$, we have to show it induces a causal tree morphism $\operatorname{Unf}(f): \operatorname{Unf}(\mathcal{E}) \longrightarrow \operatorname{Unf}\left(\mathcal{E}^{\prime}\right)$. Each path in $\operatorname{Pconf}(\mathcal{E})$ is given by a sequence of priconfigurations $\emptyset \prec X_{1} \prec X_{2} \prec \ldots \prec X_{n}$, which is mapped on a sequence $f(\emptyset)=\emptyset \prec$ $f\left(X_{1}\right) \prec f\left(X_{2}\right) \prec \ldots \prec f\left(X_{n}\right)$, defining a path labelled in the same way (possibly with more causes) because $\prec, \leq$ and labelling are preserved. The glueing increases because $f$ is a function.

\section{Theorem 5.1 Unf has a lefl adjoint L.}

Proof. The action of $L$ on the objects of CT is rather simple: each arc of the tree (which can be identified via a pair $\langle j, k\rangle$, where $j$ belongs to the inclexing set of the tree and $k$ is a natural number expressing the deptl of the arc in the tree; note that many pairs correspond to a single arc because of glueing) is an event; the back pointers along each path induces the causality ordering of the ples, the total ordering of the path induces the priority ordering and two arcs on different paths are always in conflict.

Given a tree morphism $f: C \longrightarrow C^{\prime}$, the corresponding morphism on ples $\mathbf{L}(f)$ : $\mathbf{L}(C) \longrightarrow \mathbf{L}\left(C^{\prime}\right)$ is defined by $\langle j, k\rangle \mapsto\langle f(j), k\rangle$ (the triangular inequality guarantees that $\mathrm{L}(f)$ is well defined). The reader can check that this morphism is an arrow of PLES.

In order to show that the just decribed constructions define a left adjoint $\mathbf{L}$, we build a universal arrow for eaclı causal tree $C$, i.e., a tree morphism

$$
C \longrightarrow \operatorname{Unf}(\mathbf{L}(C))
$$

which factors bijectively and naturally all morphisms $\mathbf{L}(C) \longrightarrow \mathcal{E}$, for any prioritized event structure $\mathcal{E}$.

Since each finite configuration $X \in \operatorname{Pconf}(\mathrm{L}(C))$ is of the form $\{\langle j, 0\rangle,\langle j ; 1\rangle, \ldots,\langle j, n\rangle\}$, with $n<\left|e_{C}(j)\right|$ (for $n=-1$ we get the empty configuration), and since

$$
\{\langle j, 0\rangle,\langle j, 1\rangle, \ldots,\langle j, n-1\rangle\} \prec\{\langle j, 0\rangle,\langle j, 1\rangle, \ldots,\langle j, n\rangle\},
$$

we can define the universal arrow $C \longrightarrow \operatorname{Unf}(\mathrm{L}(C))$ as

$$
j \mapsto \emptyset,\{\langle j, 0\rangle\},\{\langle j, 0\rangle,\langle j, 1\rangle\}, \ldots,\left\{\langle j, 0\rangle,\langle j, 1\rangle, \ldots\left\langle j,\left|e_{C}(j)\right|-1\right\rangle\right\} .
$$

For any morphism $f: C \longrightarrow \operatorname{Unf}(\mathcal{E})$, if

$$
j \mapsto \emptyset \prec X_{1}^{j} \prec X_{2}^{j} \prec \ldots \prec X_{n}^{j}
$$

then we define the associated morphism $g: \mathrm{L}(C) \longrightarrow \mathcal{E}$ by letting

$$
\langle j, k\rangle \mapsto X_{k}^{j} \backslash X_{k-1}^{j} \text {. }
$$

It is then easy to show that the composition with the universal arrow induces a natural bijection.

Note that $\mathrm{L}$ is an inclusion. Thus, 
Theorem 5.2 CT is a coreflective subcatcgory of PLES.

\section{Semantic Priority Operators}

We claim that the new model of prioritized labelled event structures can be used quite profitably for giving denotational semantics to process algebras enriched with operators of priority. Furthermore, with the "equivalent" model of causal trees we equip these prioritized constructs with an incluctive definition in denotational (and operational) styles.

For the sake of brevity, we consider the semantics of a prioritized nondeterministic choice and of a prioritized parallel composition, ${ }^{1}$ only. We introduce a family of semantic operators of the form $\mathcal{E}_{0}+_{\mathcal{R}} \mathcal{E}_{1}$ and $\mathcal{E}_{0} \|_{\mathcal{R}} \mathcal{E}_{1}$, respectively. The index $\mathcal{R}$ is a partial ordering on the labels of events that induces a priority relation $\ll$ on the events of $\mathcal{E}_{0}$ and $\mathcal{E}_{1}$. Note that a notion of "dynamic" priority can be expressed easily through the indexing. When $\mathcal{R}=\emptyset$, the standard choice and parallel operators are recovered. This approach is in the line of [Jan87, CH89, BK92]; a priority determined by textual position, similar to the one used in PRIALT of occam [JG88, Cam89], can be accomodated smoothly.

The operations $\mathcal{E}_{0} t_{\Re} \mathcal{E}_{1}$ and $\mathcal{E}_{0} \|_{\mathcal{R}} \mathcal{E}_{1}$ over ples's are defined as follows, assuming $\mathcal{E}_{i}=\left\langle E_{i}, \leq_{i}, \#{ }_{i}, \ll_{i}, \ell_{i}\right\rangle, i=0,1$, with $E_{0} \cap E_{1}=\emptyset$ :

pri-sum: $\mathcal{E}_{0}+\kappa \mathcal{E}_{1} \stackrel{\text { der }}{=}\left\langle E_{0} \cup E_{1}, \leq_{0} \cup \leq_{1}, \#, \ll, \ell_{1} \cup \ell_{2}\right\rangle$ where

$$
\begin{aligned}
& \#=\#_{0} \cup \#_{1} \cup\left(E_{0} \times E_{1}\right) \cup\left(E_{1} \times E_{0}\right) \\
& \ll=\ll_{0} \cup \ll_{1} \cup\left\{\left\langle\epsilon, e^{\prime}\right\rangle \mid\left\langle\ell_{i}(e), \ell_{i+1 \bmod 2}\left(e^{\prime}\right)\right\rangle \in \mathcal{R}, i=0,1\right\} .
\end{aligned}
$$

pri-parallel: $\mathcal{E}_{0} \|_{\kappa} \mathcal{E}_{1} \stackrel{\text { def }}{=}\left\langle E_{0} \cup E_{1}, \leq_{0} \cup \leq_{1}, \#, \ll, \ell_{0} \cup \ell_{1}\right\rangle$, where

$$
\begin{aligned}
& \#=\#_{0} \cup \#_{1} \\
& \ll=\ll_{0} \cup \ll_{1} \cup\left\{\left\langle e, e^{\prime}\right\rangle \mid\left\langle\ell_{i}(e), \ell_{i+1 \bmod 2}\left(e^{\prime}\right)\right\rangle \in \mathcal{R}, i=0,1\right\} .
\end{aligned}
$$

Moreover, prioritized choice and parallel operators can be defined directly over causal trees, thus facilitating the definition of an operational sos semantics for a concurrent language equipped with priority (see [DDS9] for other usual semantic operations).

Let $\mathcal{R} \subseteq \Sigma \times \Sigma$ be a priority relation; let $I$ and $J$ be disjoint sets of indexes; and let $T=\sum_{I}\left\langle\sigma_{i}, K_{i}\right\rangle \cdot T_{i}$ and $U=\sum_{J}\left\langle\nu_{j}, H_{j}\right\rangle \cdot U_{j}$ be causal trees, represented in algebraic style. Finally, let $I^{\prime}=\left\{i \in I \mid \neg \exists j \in J .\left\langle\nu_{j}, \sigma_{i}\right\rangle \in \mathcal{R}\right.$ and $J^{\prime}=\left\{j \in J \mid \neg \exists i \in I .\left\langle\sigma_{i}, \nu_{j}\right\rangle \in\right.$ $\mathcal{R}\}$. Then, the operators of prioritized nondeterministic choice, $\oplus \boldsymbol{R}$, and of asynchronous parallel composition, $\|_{R}$, are defined as follows.

$$
T \oplus \mathcal{R} U=\sum_{I^{\prime}}\left\langle\sigma_{i}, K_{i}\right\rangle \cdot T_{i}+\sum_{J^{\prime}}\left\langle\nu_{j}, H_{j}\right\rangle \cdot U_{j}
$$

and

$$
T \backslash_{\mathcal{R}} U=\sum_{I^{\prime}}\left(\sigma_{i}, K_{i}\right\rangle \cdot\left(T_{i} \backslash_{\mathcal{R}}(\delta(U) \backslash A)\right)+\sum_{J^{\prime}}\left(\nu_{j}, H_{j}\right\rangle \cdot\left((\delta(T) \backslash B) \bigwedge_{\mathcal{R}} U_{j}\right)
$$

where

\footnotetext{
${ }^{1}$ Communication is not considered as it is orthogonal to priority, and can be clealt with following the techniques developed for prime event structures, see, e.g., [Vaa89].
} 
- $A=\left\{\nu_{j} \mid j \in J, \exists i \in I .\left\langle\nu_{j}, \sigma_{i}\right\rangle \in \mathcal{R}\right\}$, and $B=\left\{\sigma_{i} \mid i \in I, \exists j \in J .\left\langle\sigma_{i}, \nu_{j}\right\rangle \in \mathcal{R}\right\}$,

- $\delta(T)$ increments all the causes in $T$ pointing behind its root (see [DD89] for details),

- $T \backslash A$ is the usual pruning operation over the set of labels $\langle\sigma, K\rangle$, with $\sigma \in A \subseteq \Sigma$.

Lack of space prevents us from inductively defining the operalional semantics of the two operators, in the style of [DDS9]. However, the task is easy; we just mention that the constraints expressed by sets $I^{\prime}$ and $J^{\prime}$ are reflected by negative premises in the inference rules.

The following proposition states that the above operations on ples's and on causal trees agree in a strong sense. It provides the basis for proving that the causal tree operational semantics and the ples denotational one coincide, up to strong bisimulation.

Proposition 6.1 Given two ples's $\mathcal{E}_{0}$ and $\mathcal{E}_{1}$, the following hold:

- $\operatorname{Unf}\left(\mathcal{E}_{0}\right) \oplus \mathfrak{R} \operatorname{Unf}\left(\mathcal{E}_{1}\right)$ is isomorphic to $\operatorname{Unf}\left(\mathcal{E}_{0}+\boldsymbol{R} \mathcal{E}_{1}\right)$,

- $\operatorname{Unf}\left(\mathcal{E}_{0}\right) \|_{\mathcal{R}} \operatorname{Unf}\left(\mathcal{E}_{1}\right)$ is isomorphic to $\operatorname{Unf}\left(\mathcal{E}_{0} \|_{\mathcal{R}} \mathcal{E}_{1}\right)$.

\section{Acknowledgements}

The authors would like to acknowledge the useful discussions with S. Kasangian and V. Sassone. Pierpaolo Degano was partially supported by C.N.R project "Progetto finalizzato sistemi informatici e calcolo parallelo" and by project MASK SC1-CT92-00776, founded by C.E.C.. Roberto Gorrieri was partially supported by C.E.C. Esprit Programme Basic Research Action Number 6360 (BROADCAST). The authors were also partially supported by M.U.R.S.T. $40 \%$.

\section{References}

[BK92] E. Best and M. lioutny. Petri net semantics of priority systems. Theoretical Computer Science, 96:175-215, 1992.

[Cam89] J. Camilleri. An operational semantics for occam. International Journal of Parallel Programming, 18(5), 1989.

[CH89] R. Cleaveland and M. Hennessy. Priorities in process algebras. In Proc. LICS '89, pages 193-202, Edinburgh, 1989.

[DD89] Ph. Darondeau and P. Degano. Causal trees. In Proc. 11th Int. Coll. on Automata and Languages ICALP, number 372 in LNCS, pages 234-248. SpringerVerlag, 1989.

[DD90] Ph. Darondeau and P. Degano. Causal trees: Interleaving + causality. In Proc. 18th École de Printemps sur la Semantique de Parallelism, number 469 in LNCS, pages 239-255. Springer-Verlag, 1990.

[DDM90] P. Degano, R. De Nicola, and U. Montanari. A partial ordering semantics for CCS. Theoretical Computer Science, 75:223-262, 1990. 
[Hoa.85] C.A.R. Hoare. Communicaling Sequential Processes. Prentice Hall, Englewood Clifls, NJ, 1985.

[Jan87] R. Janicli. A formal semantics of concurrent systems with a priority relation. Acla Informatica, 24:33-55, 1987.

[JGS8] G. Jones and M. Goldsmith. Programming in occam-2. Prentice-Hall, Englewood Cliffs, N.J, 1988.

[JK91] R. Janicli and M. Koutny. Invariant semantics with inhibitor arcs. In Proc. CONCUR '9l, number' 527 in LNCS, pages 317-331. Springer-Verlag, 1991.

[KLP90] S. Kasangian, A. Labella, and A. Pettorossi. Observers, experiments and agents: a comprehensive approach to concurrency. In I. Guessarian, editor, Semantics of Systems of Concurrenl. Processes, number 469 in LNCS. Springer-Verlag, 1990.

[Law74] F.W. Lawvere. Melric spaces, generalized logic, and closed categories. Rendiconti Seminario Mat. e Fis. di Milano, 43:135-166, 1974.

[Mil80] R. Milner. A Calculus of Communicating Systcms. Number 92 in LNCS. Springer-Verlag, 1980.

[NPW81] M. Nielsen, G. Plotkin, and G. Winskel. Petri nets, event structures, and domains, part I. Theoreticul Computer Science, 13:85-108, 1981.

[Pra86] V.R. Pratt. Modeling concurrency with partial orders. International Journal of Parallel Progranming, 15(1):33-71, 1986.

[Roz90] B. Rozoy. On distributed languages and models for distributed computations. In I. Guessarian, editor, Semanlics of Systems of Concurrent Processes, number 469 in LNCS, pages 434-456. Springer-Verlag, 1990.

[RTS8] A. Rabinovich and B.A. Trakhtenbrot. Behavior structures and nets. Fundamenla Informaticae, 11(4):35i-404, 1988.

[SNW] V. Sassone, M. Nielsen, and G. Winskel. A hierarchy of models for concurrency. Technical report. Draft.

[Vaa89] F.W. Vaandrager. A simple definition for parallel composition of prime event structures. Technical Report CS-1R 8903, CWI, Amsterdam, 1989.

[Wal82] R.F.C. Walters. Sheaves on sites as Cauchy-complete categories. Journal of Pure and Applied Algebra, 24:95-102, 1982.

[Win87] G. Winskel. Event structures. In Petri Nets: Applications and Relationships to Other Models of Concurrency, Advances in Petri Nets 1986, Part II, number 255 in LNCS, pages 325-392. Springer-Verlag, 1987. 